MMCA-97 Conference, pp. 79-83

R. Čiegis (Ed)

(C) 1997 Vilniaus Gedimino technikos universitetas

\title{
POLYCONVOLUTION FORMATION OF FINITE INTEGRAL TRANSFORMATIONS
}

\author{
V.A. KAKICHEV \\ Novgorod State University, Dept. of Theoretical Physics \\ Novgorod, 173003 RUSSIA \\ e-mail: teorfis@lan.novsu.ac.ru
}

\section{SECTION 1}

We introduce necessary notations: let $P$ is subset of set of integers; $\tilde{U}_{\alpha}(X)$, $\tilde{U}_{\beta}(Y), \tilde{U}_{\gamma}(Z)$ are linear spaces of functions of kind:

$$
\begin{aligned}
a(x) & =\sum_{n \in P} a_{n} \alpha_{n}(x), & & x \in X, \\
b(y) & =\sum_{n \in P} b_{n} \beta_{n}(y), & & y \in Y, \\
c(z) & =\sum_{n \in P} c_{n} \gamma_{n}(z), & & z \in Z,
\end{aligned}
$$

where

$$
\bar{\alpha}(x)=\left\{\alpha_{n}(x)\right\}_{n \in P}, \bar{\beta}(y)=\left\{\beta_{n}(y)\right\}_{n \in P}, \bar{\gamma}(z)=\left\{\gamma_{n}(z)\right\}_{n \in P}
$$

are basic systems of functions. $V(P)$ is set of factors:

$$
\bar{a}=\left\{a_{n}\right\}_{n \in P}, \bar{b}=\left\{b_{n}\right\}_{n \in P}, \bar{c}=\left\{c_{n}\right\}_{n \in P}
$$

such, that :

a) at $\bar{a}, \bar{b}$, and $\bar{c}$ from $V(P)$ all expansions (1) make sense;

b) $V(P)$ - forms the algebra relatively by coordinate of multiplication:

$$
\bar{a} \cdot \bar{b}=\left\{a_{n} \cdot b_{n}\right\}_{n \in P} \in V(P), \forall \bar{a}, \bar{b} \in V(P) .
$$

Hence, the linear operators are defined as:

$$
A: V(P) \rightarrow \widetilde{U}_{\alpha}(X), B: V(P) \rightarrow \widetilde{U}_{\beta}(Y), C: V(P) \rightarrow \widetilde{U}_{\gamma}(Z),
$$


associating to elements (3) from $V(P)$ functions: $a(x) \in \widetilde{U}_{\alpha}(X), b(y) \in$ $\widetilde{U}_{\beta}(Y), c(z) \in \widetilde{U}_{\gamma}(Z)$ with help series $(1)$.

Assuming:

$$
A V(P)=U_{\alpha}(X), B V(P)=U_{\beta}(Y), C V(P)=U_{\gamma}(Z)
$$

we shall suppose that inverse operators $A^{-1}, B^{-1}$ and $C^{-1}$ are defines, which bijectively mapping spaces $U_{\alpha}(X), U_{\beta}(Y)$ and $U_{\gamma}(Z)$ respectively on $V(P)$ and by this the linear functionals are given:

$$
\left(a(x), \alpha_{n}(x)\right)=a_{n},\left(b(y), \beta_{n}(y)\right)=b_{n},\left(c(z), \gamma_{n}(z)\right)=c_{n}, n \in P .
$$

The combinations of two types of basic systems are considered below: a) orthonormal systems (2) with weight functions $u(x), v(y)$ and $w(z)$ respectively and consequently

a)

$$
\begin{aligned}
a_{n}=\int_{X} u(x) a(x) \alpha_{n}(x) d x, b_{n} & =\int_{Y} v(y) b(y) \beta_{n}(y) d y, \\
c_{n} & =\int_{Z} w(z) c(z) \gamma_{n}(z) d z,
\end{aligned}
$$

b)

$$
\begin{aligned}
& \alpha_{n}(x)=x^{n}, \beta_{n}(y)=y^{n}, \gamma_{n}(z)=z^{n}, \text { so } \\
& a_{n}=\frac{1}{2 \pi i} \int_{L_{X}} a(x) \frac{d x}{x^{n+1}}, b_{n}=\frac{1}{2 \pi i} \int_{L_{Y}} b(y) \frac{d y}{y^{n+1}}, c_{n}=\frac{1}{2 \pi i} \int_{L_{Z}} c(z) \frac{d z}{z^{n+1}},
\end{aligned}
$$

where $L_{X} \subset X, L_{Y} \subset Y, L_{Z} \subset Z$ are smooth closed contours, enveloping origin.

Let $\bar{\rho}$ is fixed vector from $V(P)$. Polyconvolution which generated by operators $C, A^{-1}, B^{-1}$ and weight $\bar{\rho}$ we shall define as the sum of series

$$
\begin{array}{r}
c(z)=\sum_{n \in P} \rho_{n} a_{n} b_{n} \gamma_{n}(z)=[C(\bar{\rho} \cdot \bar{a} \cdot \bar{b})](z)= \\
\left\{C\left[\bar{\rho} \cdot\left(A^{-1} a(x)\right) \cdot\left(B^{-1} b(y)\right)\right]\right\}(z) \equiv\left[\begin{array}{c}
\bar{\rho} \\
a * b
\end{array}\right](z) .
\end{array}
$$

Substituting functionals (8) or/and (9) in (10), determining factors $a_{n}$ and $b_{n}$, and (formally!) rearranging the order of summation and integration, we have 
three types of convolutions:

$$
c(z)=\left\{\begin{array}{l}
\int_{X} \int_{Y} u(x) v(y) \Theta(z, x, y) a(x) b(y) d x d y, \\
\frac{1}{2 \pi i} \int_{L_{X}} \int_{Y} v(y) \Theta(z, x, y) a(x) b(y) \frac{d x}{x} d y, z \in Z, \\
\frac{1}{(2 \pi i)^{2}} \int_{L_{X}} \int_{L_{Y}} \Theta(z, x, y) a(x) b(y) \frac{d x}{x} \frac{d y}{y}
\end{array}\right.
$$

kernels of which are one of four various values:

$$
\Theta(z, x, y)= \begin{cases}\sum_{n \in P} \rho_{n} \gamma_{n}(z)\left(\begin{array}{c}
\alpha_{n}(x) \beta_{n}(y) \\
x^{-n} \beta_{n}(y) \\
(x y)^{-n}
\end{array}\right) & \text { at } \gamma_{n} \neq z^{n}, \\
\sum_{n \in P} \rho_{n}\left(\frac{z}{y x}\right)^{n} & \text { at } \gamma_{n}=z^{n} .\end{cases}
$$

Thus, problem of search of explicit form polyconvolutions (11)-(12) is reduced to:

1) validity of rearrangement order of summation and integration in (10),

2) summations of series (12), which describes kernels of convolutions (11),

3) belonging of convolution (11) to $U_{\gamma}(Z)$.

\section{SECTION 2}

Now we define dual to (10)-(11) polyconvolutions. At first we some change previously introduced notations.

Let:

$P_{a}, P_{b}, P_{c}$ - are subsets of set integers;

$\tilde{V}\left(P_{a}\right), \tilde{V}\left(P_{b}\right), \tilde{V}\left(P_{c}\right)$ are linear spaces of sequences (compare with (3)):

$$
\bar{a}=\left\{a_{n}\right\}_{n \in P_{a}}, \bar{b}=\left\{b_{n}\right\}_{n \in P_{b}}, \bar{c}=\left\{c_{n}\right\}_{n \in P_{c}},
$$

such that correspondenting expansions (1) are valid, in which summation is conducted by sets $P_{a}, P_{b}$, and $P_{c}$ instead of $P$, and $X=Y=Z$. Hence, in (2) $P$ is also replaced by corresponding sets $P_{a}, P_{b}$, and $P_{c}$. In these cases instead of (1) and (2) we shall write (1') and (2').

We notate set of functions (1') as $U(X)$ such that:

1) if $a(x)=b(x)=c(x)$ from $U(X)$, then all three expansions (1') on bases (2') take place, 
2) $U(X)$ - is algebra with by coordinate multiplication :

So, linear operators are defined (compare with (5)):

$$
A^{-1}: U(X) \rightarrow \widetilde{V}\left(P_{a}\right), B^{-1}: U(X) \rightarrow \widetilde{V}\left(P_{b}\right) \quad C^{-1}: U(X) \rightarrow \widetilde{V}\left(P_{c}\right),
$$

which associated functions $a(x), b(x)$ and $c(x)$ from $U(X)$ and coefficients sequences $\left(3^{\prime}\right)$, determined by means of functionals $(8)$ or $(9)$.

Let us (compare with (6)):

$$
A^{-1} U(X)=V\left(P_{a}\right), B^{-1} U(X)=V\left(P_{b}\right), C^{-1} U(X)=V\left(P_{c}\right) .
$$

We associate to arbitrary functions $a(x), b(x)$ and fixed function $r(x)$ from $U(X)$ the product

$$
c(x)=r(x) a(x) b(x)=r(x) \sum_{p \in P_{a}} \sum_{q \in P_{b}} a_{p} b_{q} \alpha_{p}(x) \beta_{q}(x)=\sum_{n \in P_{c}} c_{n} \gamma_{n}(x) .
$$

This product defines vector $\bar{c} \in V\left(P_{c}\right)$ with help polyconvolution by vectors $\bar{a} \in V\left(P_{a}\right), \bar{b} \in V\left(P_{b}\right)$, and by weight function $r(x)$.

$$
\bar{c}=\bar{a} * \bar{b}=C^{-1}[r(x) \cdot(A \bar{a})(x) \cdot(B \bar{b})(x)]=\left\{\sum_{p \in P_{a}} \sum_{q \in P_{b}} \Theta_{n, p q} a_{p} b_{q}\right\}_{n \in P_{c}}
$$

Explicit form of kernel $\Theta_{n, p q}$ from (15) depends on structure of functionals (8) and (9), determening $C_{n}$ at $n \in P_{c}$ :

$$
\Theta_{n, p q}= \begin{cases}\int_{X} r(x) w(x) \gamma_{n}(x) \alpha_{p}(x) \beta_{q}(x) d x, & \gamma_{n} \neq x^{n} \\ \frac{1}{2 \pi i} \int_{L_{X}} r(x) \alpha_{p}(x) \beta_{q}(x) \frac{d x}{x^{n+1}}, & \gamma_{n}=x^{n} .\end{cases}
$$

Here in (16.2) was included cases, when, for example, $\beta_{q}=x^{q}$ or $\alpha_{p}=$ $x^{p}, \beta_{q}=x^{q}$.

\section{SECTION 3}

a) However is shown as on finite integral operators and inverse for its operators may find kernels (12) and (16) of polyconvolutions (11) and (15). The reverse (more strong) statement is true too: the explicit form of operators, determining polyconvolutions (11) and (15) may be reconstructed by kernels (12) and (16) structures unambigiously. Moreover, circular rearrangement of 
operators, forming polyconvolutions, results in same kernels (12) and (16) of polyconvolutions (11) and (15).

b) In present time we prepearing the article, where by present here common scheme constructed particular polyconclusions. As basic systems are used, for example :
1) $\left\{x^{n}\right\}$,
2) trigonometrical ones,
3) classical polynomials,
4) special functions.

The close questions were considered in articles[1-3].

c) About constructing of polyconvolutions of integral transformations author maked report on international conference "Boundary-value problems, special functions and fractional calculus", devoted by 90 from the date birth of academician F.D.Gakhov (Minsk, Belarus, on February 16-20,1996). On this theme you can see $[4,5]$.

\section{REFERENCES}

[1] Berezanskii Yu.M. Hypercomplex systems with discrete basis. Doklady Academy of Science, USSR, 1951, 81(3), P. 329-332. (In Russian).

[2] Kakichev V.A. The formations of convolutions determined of factorizable operators (discrete case ). Math. semulation and its applications, Novgorod, 1993, P. 52-59. (In Russian).

[3] Kakichev V.A. Bilinear kernels and convolutions of functionals. Vestnik NovSU, Ser.: Estestv. i tekhn. nauki, Novgorod, 1996, No. 3, P. 82- 86.

[4] Kakichev V.A., Thao N.X., and Hai N.T. Composition Method for Constructing Convolutions for Integral Transforms. Integral Transforms and Special Functions, 1996, No 3, P. 235-242.

[5] Kakichev V.A., Nguen Suan Thao. About one method of constructing of generalized integral convolutions. Izv. Vuzov. th., 1997, No 10. (In Russian). 